of mobile stroke unit patients achieve a good or excellent outcome (modified Rankin scale of 0-2). Additionally, $44 \%$ of stroke patients treated in the mobile stroke unit return to a pre-stroke baseline compared to $36 \%$ in the Emergency Department. There is no increase in mortality rates in the mobile stroke unit group compared to Emergency Department treatment. Symptomatic hemorrhagic transformation rate is $0 \%$ compared to $5 \%$ in the Emergency Department. In conclusion, the mobile stroke unit has demonstrated a time to treatment benefit. Additionally, there has been an increase in treatment within the 'Golden Hour'. There has been no increase in mortality or complication rates.

Disclosures H. Shownkeen: None. A. Lindstrom: None. H. Crumlett: None. M. Mohajer-Esfahani: None. D. Lumb: None. S. Graham: None.

\section{E-093 GEOGRAPHICAL VARIATION IN THE DECISION TO PROCEED WITH ENDOVASCULAR TREATMENT UNDER 'CURRENT' AND 'IDEAL' SETTINGS}

${ }^{1} \mathrm{~N}$ Kashani ${ }^{*},{ }^{2} \mathrm{~J}$ Ospel, ${ }^{1} \mathrm{M}$ Almekhlafi, ${ }^{1} \mathrm{~A}$ Wilson, ${ }^{3} \mathrm{~A}$ Rabinstein, ${ }^{4} \mathrm{~W}$ Kunz, ${ }^{5} \mathrm{~B}$ Campbell, ${ }^{1} \mathrm{M}$ Foss, ${ }^{6} \mathrm{~F}$ Turjman, ${ }^{7} \mathrm{U}$ Fischer, ${ }^{8} \mathrm{~B}$ Baxter, ${ }^{9} \mathrm{P}$ Sylaia, ${ }^{10} \mathrm{~S}$ Yoshimura, ${ }^{11} \mathrm{~J} \mathrm{Heo,}{ }^{12} \mathrm{~B}$ Kim, ${ }^{1} \mathrm{M}$ Hill, ${ }^{13} \mathrm{G}$ Saposnik, ${ }^{1} \mathrm{~B}$ Menon, ${ }^{1} \mathrm{M}$ Goyal. ${ }^{1}$ Radiology, University of Calgary, Calgary, $A B$, Canada; ${ }^{2}$ Radiology, University Hospital Basel, Basel, Switzerland; ${ }^{3}$ Radiology, Mayo Clinic Rochester, rochester, OR; ${ }^{4}$ Radiology, University Hospital Munich, Munich, Germany; ${ }^{5}$ Neurology, Royal Melbourne Hospital, University of Melbourne, Melbourne, Australia; ${ }^{6}$ Radiology, University of Calgary, Lyon, France; ${ }^{7}$ Radiology, University Hospital Bern (Inselspital), Bern, Switzerland; ${ }^{8}$ Radiology, Erlanger Hospital Chattanooga, Chattanooga, KS; ${ }^{9}$ Radiology, Sree Chitra Tirunal Institute for Medical Sciences and Technology, Thiruvananthapuram, India; ${ }^{10}$ Neurosurgery, Hyogo College of Medicine, Hyogo, Japan; ${ }^{11}$ Radiology, Yonsei University, Seoul, Korea, republic of; ${ }^{12}$ Radiology, St. Mary's Hospital Seoul, Seoul, Korea, republic of; ${ }^{13}$ Radiology, University of Toronto, Calgary, ON, Canada

10.1136/neurintsurg-2019-SNIS. 168
Background In light of multiple trials showing efficacy of mechanical thrombectomy with highest level of supporting evidence, limited information is available on how treating physicians around the world make decisions in real-life regarding patient selection for (EVT). Regional differences exists where for the same patient presentation and attributes, the decision to treat endovascularly could differ based on local practice and resource settings. In this study we seeked to determine the effect of regional and country specific factors on endovascular treatment and how those decisions could differ in a simulated 'ideal' setting if resources were not to be constrained.

Methods We conducted a multidisciplinary survey of Neurosurgeons, Neurointerventional Radiologists, Neurologists, and other clinicians involved in acute stroke care around the world in an effort to understand prevailing approaches in management of stroke using a case-based approach $(n=603$ from 38 countries). The participants were presented with 10 randomly assigned cases with varying patient characteristics. Respondents were asked which treatment they would selected for each patient in their 'current' practice setting versus 'ideal' conditions. The rate of EVT selection was calculated and compared across different world countries. The propensity to add EVT under 'ideal' conditions were compared across geographic regions taking into account physician age, experience, specialty, and academic practice setting.

Results In the case scenarios $75.5 \%$ of the respondents proceeded with EVT under 'current' practice setting and were stratified into 7 geographic regions (6070 responses). Regions with relatively lower than average positive rates in proceeding with EVT were South America $(58 \% \mathrm{n}=24)$ and South Asia $(60.5 \%$ of $n=24)$, compared to $78 \%$ global. Average age of the treating clinicians in South America was 37.3 versus 44.8 global average $(H=373.6 ; p<0.001)$ which reflected number of years in practice 9.4 vs. 14.2 yrs
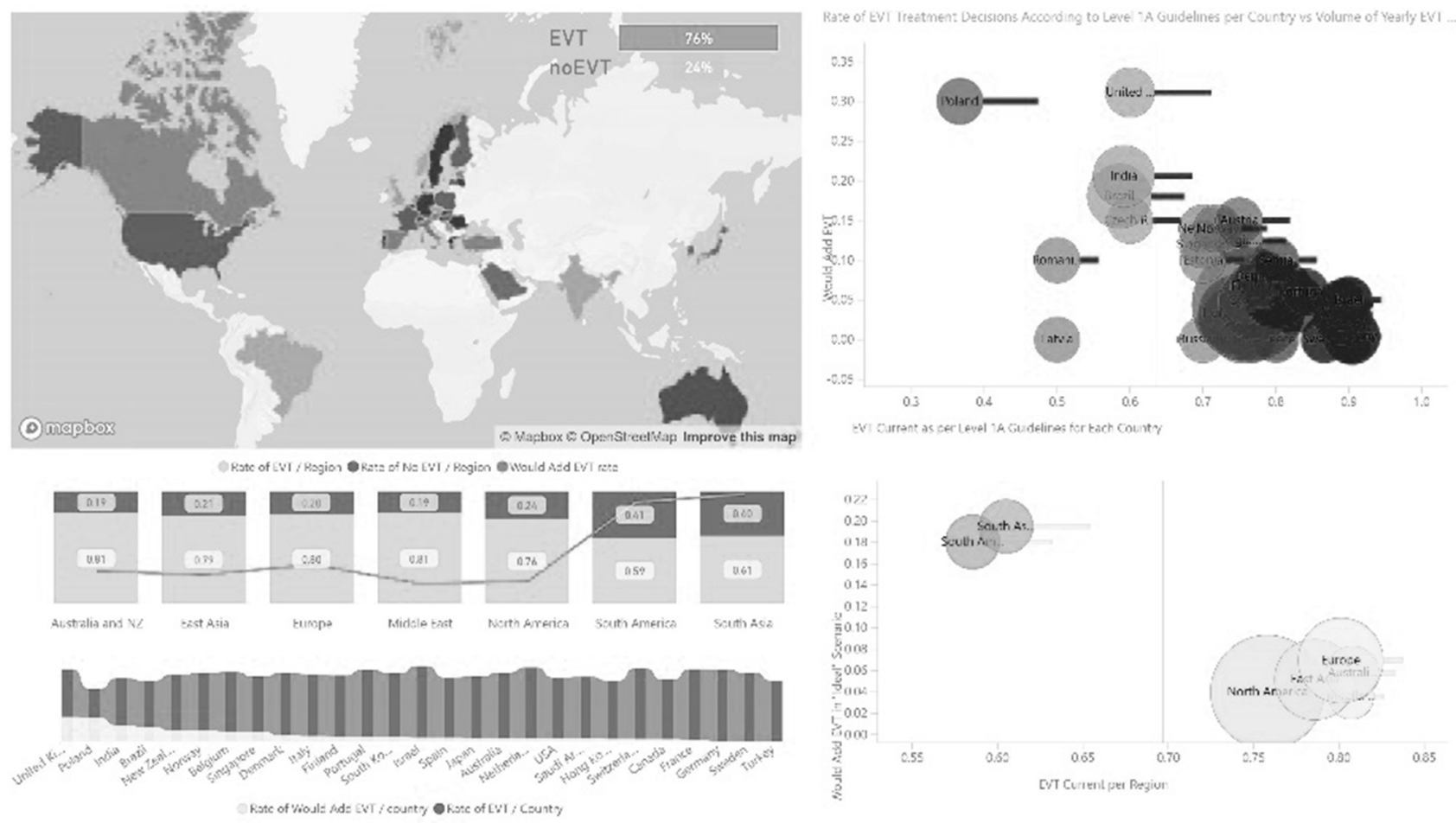
average $(\mathrm{H}=637.1 ; \mathrm{p}<0.001)$. When considering an 'ideal' setting, clinicians in South America $18 \%$ and South Asia $19.5 \%$ were most likely to add EVT versus the $6.9 \%$ global average.

Conclusion In the selection of patients to undergo endovascular treatment, there exists a significant difference in rate of EVT procedures performed across countries and regions in the world reflecting local resources and practice settings. The decision to proceed with EVT reflects factors such as center volumes and clinician age $\&$ experience as contributing factors with younger clinicians in centers with lower volumes being more likely to consider EVT in an 'ideal' setting.

Disclosures N. Kashani: None. J. Ospel: None. M. Almekhlafi: None. A. Wilson: None. A. Rabinstein: None. W. Kunz: None. B. Campbell: None. M. Foss: None. F. Turjman: None. U. Fischer: None. B. Baxter: None. P. Sylaia: None. S. Yoshimura: None. J. Heo: None. B. Kim: None. M. Hill: None. G. Saposnik: None. B. Menon: None. M. Goyal: None.

\section{E-094 EFFECT OF DEFINITION AND METHODS ON ESTIMATES OF PREVALENCE OF LARGE VESSEL OCCLUSION IN ACUTE ISCHEMIC STROKE: SYSTEMATIC REVIEW}

${ }^{1} \mathrm{M}$ Waqas*, ${ }^{2} \mathrm{~A}$ Rai, ${ }^{1} \mathrm{~K}$ Vakharia, 'A Siddiqui. ${ }^{1}$ UB Neurosurgery, Buffalo, NY; ${ }^{2}$ Radiology, West Virginia University Hospital, Morgantown, WV

\subsection{6/neurintsurg-2019-SNIS.169}

Background An accurate estimate of large vessel occlusion (LVO) incidence is critical for planning stroke systems of care and approximating workforce requirements. The purpose of this systematic review was to estimate LVO prevalence among acute ischemic stroke (AIS) patients with emphasis on definitions and methods used by different studies.

Methods Using Medical Subject Headings terms, EMBASE, PubMed, Web of Science, Google Scholar, and the Cochrane Library were searched for prevalence of LVO and AIS from 2000-2018. All articles in the English literature describing the frequency of LVO among AIS patients were included in this review. Studies without consecutive recruitment or without confirmation of LVO with computed tomography angiography or magnetic resonance angiography were excluded. Studies were grouped based on similarities in LVO definition and methods.

Results Eighteen records met the eligibility criteria. Five studies presented population-based estimates and 13 provided single-hospital experiences (5 prospective, 8 retrospective). The AIS denominator (number of all AIS) from which LVO rates were generated was variable. Nine different definitions were used, based on occlusion site. The prevalence of LVO among suspected AIS patients ranged from 13-46\%. The overall prevalence was 29.1\% (95\% CI, 21.5-36.77). Pooled prevalence of LVO among suspected AIS patients in population-based studies was $27.10 \%$ (95\% CI 19.46-34.74); for studies defining AIS based on magnetic resonance imaging, it was $21.49 \%$ (95\% CI 13.8-29.1).

Conclusion LVO definition and AIS denominator greatly influence estimates of LVO prevalence among AIS patients. Population-based studies with standardized methods must be conducted to provide data for planning and developing stroke systems of care.
Disclosures M. Waqas: None. A. Rai: 2; C; Medtronic, Cerenovus. K. Vakharia: None. A. Siddiqui: 1; C; Co-investigator NIH/NINDS 1R01NS091075. 2; C; Amnis Therapeutics, Boston Scientific, Canon Medical Systems USA Inc, Cerebrotech Medical Systems, Cerenovus, Corindus Inc., Endostream Medical Ltd, Guidepoint Global Consulting, Imperative Care, Integra LifeSciences Corp, Medtronic, MicroVention, Q’Apel Medical Inc, Rapid Medical, Rebound Therapeutics Corp., Serenity Medical Inc, Silk Road Medical, StimMed, Stryker, Three Rivers Medical, VasSol, W.L. Gore \& Associates. 4; C; Amnis Therapeutics, Apama Medical, Blink TBI Inc, Buffalo Technology Partners Inc, Cardinal Consultants, Cerebrotech Medical Systems, Cognition Medical, Endostream Medical Ltd, Imperative Care, International Medical Distribution Partners, Neurovascular Diagnostics Inc, Q'Apel Medical Inc, Rebound Therapeutics Corp, Rist Neurovascular In, Serenity Medical, Silk Road Medical, StimMed, Synchron, Three Rivers Medical Inc, Viseon Spine.

\section{E-095 BGC PROTECTED ADAPT REDUCED RISK OF DISTAL EMBOLIZATION: AN IN VITRO STUDY}

R Arslanian*, M Marosfoi, C Raskett, MJ Gounis, AS Puri, J Chueh. New England Center for Stroke Research, UMass Medical School, Worcester, MA

10.1136/neurintsurg-2019-SNIS. 170

Introduction Recent studies have demonstrated that a direct aspiration first pass technique (ADAPT) for the treatment of acute ischemic stroke may be effective and safe. In this study, we sought to optimize the ADAPT technique by incorporating an adjunctive device such as a balloon guide catheter (BGC). The impact of diameter of the aspiration catheter as well as use of BGC on risk of distal embolization and rate of first pass success was evaluated.

Methods Mechanical thrombectomy was simulated in an invitro Circle of Willis model. To create a challenging occlusive burden in the MCA, a $20 \mathrm{~mm}$ long clot analog was prepared. Direct aspiration was applied via an 0.068 distal access catheter (DAC, Cat 7, Stryker Neurovascular, group: ADAPT068), a large 0.074' DAC (AXS Vecta 074, Stryker Neurovascular, group: ADAPT074) or 0.068 ' DAC with a BGC (FlowGate 2 Balloon Guide Catheter) placed at the cervical ICA (group: ADAPT068-BGC). In both the ADAPT068 and 074 groups, a long 0.091' sheath was used in conjunction with the DAC. The pump was turned on before the aspiration catheter was advanced proximal to the clot. Each run was assigned a TICI score to assess degree of recanalization. Ten replicates were performed per group, and the maximum thrombectomy attempt was limited to three per run. Distal emboli greater than $200 \mu \mathrm{m}$ were collected and measured using the Coulter Principle.

Results The lowest number of distal emboli was observed when BGC was used in the ADAPT068 group. Collection of visible emboli greater than $1000 \mu \mathrm{m}$ yielded one particle from both the ADAPT068 and ADAPT068-BGC treatment groups, while ADAPT074 followed with four particles (average size $2.25 \mathrm{~mm}$ ). A total of 34 emboli sized between $200-1000 \mu \mathrm{m}$ were collected from the ADAPT068 group, and the number of emboli was reduced by more than $50 \%$ when a BGC was employed in the ADAPT068-BGC group $(\mathrm{P}>0.05)$. The highest rate of first pass recanalization was observed in the 